\title{
Perfil epidemiológico dos casos notificados de sífilis congênita no município de Porto Velho entre os anos de 2010 a 2020
}

\author{
Epidemiological profile of reported cases of congenital syphilis in the municipality of Porto \\ Velho between the years 2010 to 2020
}
Perfil epidemiológico de los casos notificados de sífilis congénita en la ciudad de Porto Velho entre 2010 y 2020

João Gabriel Muniz Kisner ${ }^{*}$, Gabriela Andriolo Dickow ${ }^{1}$, Guilherme Rosa Carvalho ${ }^{1}$, Janderson Cotinguiba Santos ${ }^{1}$, João Pedro Becker de Melo e Silva1 ${ }^{1}$, Katarina Closs Fonseca Valente ${ }^{1}$, Maria Eduarda Rigotti ${ }^{1}$, Rodrigo René Bucar Durlacher ${ }^{1}$, Saymon Albuquerque ${ }^{1}$, Arlindo Gonzaga Branco Junior ${ }^{1,2}$.

\section{RESUMO}

Objetivo: Analisar os casos de sífilis congênita ocorridos no município de Porto Velho (RO), no período entre 2010 e 2020, considerando-se o perfil sociodemográficos mães e dos casos notificados pelo município. Métodos: Estudo baseado nos casos notificados no banco de dados da plataforma online disponível no Sistema de Informações de Agravos de Notificações (SINAN) do Ministério de Saúde (MS). Resultados: Obteve-se um aumento entre 2010 a 2018, passando de 7 casos notificados para 87, e um declínio nos anos de 2019 e 2020, diminuindo de 87 para 26 casos notificados. Sociodemograficamente, a faixa etária de 20 a 29 anos foi a mais suscetível, com baixa escolaridade (42,2\% possuem até o ensino fundamental), $79,2 \%$ da cor parda, 75,2 realizaram o pré-natal e $64,2 \%$ não realizaram o tratamento adequado. Conclusão: A detecção tardia aliada com a frequência da transmissão vertical da doença, apesar do acesso ao pré-natal, sugere que exista uma maior integração dos serviços de saúde envolvidos na prevenção da sífilis congênita.

Palavras-chave: Sífilis congênita, Transmissão vertical, Doenças sexualmente transmissíveis, Porto Velho.

\begin{abstract}
Objective: To analyze the cases of congenital syphilis that occurred in the city of Porto Velho (RO), between 2010 and 2020, considering the sociodemographic profile of mothers and the cases reported by the city. Methods: Study based on cases reported in the online platform database available in the Notifiable Diseases Information System (SINAN) of the Ministry of Health (MS). Results: There was an increase between 2010 and 2018, from 7 reported cases to 87, and a decline in 2019 and 2020, decreasing from 87 to 26 reported cases. Sociodemographically, the age group from 20 to 29 years old was the most susceptible, with low education ( $42.2 \%$ have up to elementary school), $79.2 \%$ of mixed race, 75.2 had prenatal care and $64.2 \%$ did not undergo the proper treatment. Conclusion: Late detection combined with the frequency of vertical transmission of the disease, despite access to prenatal care, suggest that there is a greater integration of health services involved in the prevention of congenital syphilis.
\end{abstract}

Keywords: Congenital syphilis, Vertical transmission, Sexually transmitted diseases, Porto Velho.

${ }^{1}$ Centro Universitário São Lucas (UNISL), Porto Velho - RO. *E-mail: joaogabrielpvh99@gmail.com

2 Universidade Federal de Rondônia (UNIR), Porto Velho - RO. 


\section{RESUMEN}

Objetivo: Analizar los casos de sífilis congénita ocurridos en la ciudad de Porto Velho (RO), entre 2010 y 2020, considerando el perfil sociodemográfico de las madres y los casos reportados por la ciudad. Métodos: Estudio basado en casos reportados en la base de datos de la plataforma en línea disponible en el Sistema de Información de Enfermedades de Notificación (SINAN) del Ministerio de Salud (MS). Resultados: Hubo un aumento entre 2010 y 2018, de 7 casos reportados a 87, y una disminución en los años 2019 y 2020 , disminuyendo de 87 a 26 casos reportados. Sociodemográficamente, el grupo de edad de 20 a 29 años fue el más susceptible, con baja escolaridad (42,2\% tiene hasta la primaria), 79,2\% mestizos, 75,2 tenían atención prenatal y $64,2 \%$ no se sometieron al tratamiento adecuado. Conclusión: la detección tardía combinada con la frecuencia de transmisión vertical de la enfermedad, a pesar del acceso a la atención prenatal, sugieren que existe una mayor integración de los servicios de salud involucrados en la prevención de la sífilis congénita.

Palabras clave: Sífilis congénita, Transmisión vertical, Enfermedades de transmisión sexual, Porto Velho.

\section{INTRODUÇÃO}

A sífilis uma doença infectocongiosa sistêmica, cuja principal via de transmissão é a sexual e pela transmissão vertical, conhecida como sífilis congênita, este tipo de transmissão pode ocorrer intraútero ou durante a passagem do bebê com lesões ativas no canal do parto (BRASIL, 2019; SUTO CSS, et al., 2016). A transmissão vertical de sífilis em mulheres é influenciada pelo estágio da sífilis e pela duração da exposição fetal, na fase primária e secundária a transmissão é maior, em torno de 70 a 100\% (BRASIL, 2019).

Esta infecção é conhecida há mais de 500 anos, sendo portando um antigo problema de saúde pública, apesar da existência de medidas de prevenção e de tratamento acessíveis e eficazes (WHO, 2007). A doença é causada por uma bactéria espiroqueta chamada de Treponema pallidium, descoberta em 1905, sua principal via de transmissão é a sexual, porém pode ser transmitida por transfusão sanguínea e via placentária (DOMINGUES RM, 2016; RODRIGUES AR, 2016).

A Organização Pan Americana de Saúde (OPAS) e o Fundo das Nações Unidas para a Infância (UNICEF) no ano de 2010 determinou metas para redução de casos de sífilis congênita na América Latina para 0,5 casos para 1.000 nascidos vivos até 2015. No entanto, dados brasileiros mais recentes, expostos no Boletim Epidemiológico de Sífilis de 2016, apontam que as taxas de incidência e mortalidade infantil causada pela sífilis aumentaram no período estabelecido, ou seja, o Brasil demonstrou um insucesso no controle dessa doença (ANDRADE ALMB, et al., 2018).

Após a descoberta da penicilina, década de 1940, foi observada uma acentuada queda na incidência da enfermidade. Entretanto, apesar das facilidades para se obter o diagnóstico e do tratamento de baixo custo, além de ser oferecido nas redes básicas do Sistema Único de Saúde, o número de casos notificados aumentou nas últimas décadas, no ano de 2018 foram notificados no SINAN 158.051 casos (78,8 casos por 100.000 habitantes) (DOMINGUES RM, 2016; BRASIL 2019).

A infecção pela sífilis é dividida em estágios, estes são delimitados pelos achados clínicos, eles orientam o tratamento e monitoramento do paciente. As divisões da sífilis são: primária, secundária, latente (recente e tardia) e terciária. A maioria dos diagnósticos em gestantes ocorre no estágio de sífilis latente, esta fase é assintomática, ou seja, a maior parcelo dos casos é diagnosticado por meio dos testes preconizados durante o pré-natal e o parto. Durante a gestação, esta doença pode acarretar aborto, natimorto, parto prematuro, morte neonatal e manifestações congênitas precoces ou tardias, sendo essencial o diagnóstico de sífilis durante a realização do pré-natal (BRASIL, 2019; BRASIL 2020).

O Brasil, por ano, 50 mil parturientes possuem indícios de infecção por sífilis, com isso entre as metas do Pacto pela Saúde está o controle de doenças gestacionais (LAFETÁ KR, et al, 2016). A partir disso, a Rede Cegonha, programa do Ministério da Saúde, é utilizado como promoção do diagnóstico precoce, por meio da realização de testes de triagem para gestantes e parceiros sexuais (BRASIL, 2016). Ademais, é de extrema necessidade o tratamento dos parceiros de gestantes infetadas, com a finalidade de evitar uma reinfecção (LAGO EG, 2016). 
É importante salientar que não existe uma avaliação específica para a determinação precisa do diagnóstico de sífilis na criança. À vista disso, a avaliação inicial da criança requer a combinação da história de sífilis da gestante, quanto ao tratamento e seguimento da gestação, sinais e sintomas clínicos da criança e resultados dos exames laboratoriais da criança comparado com o da mãe (BRASIL, 2019). Isso se deve ao fato de que cerca de 60 a 90\% dos neonatos portadores de sífilis congênita desenvolvem a forma assintomática da doença ao nascimento e, mesmo aqueles com alguma expressão clínica, as manifestações são, geralmente, reduzidas ou inespecíficas (BRASIL, 2019). As manifestações clínicas da sífilis congênita tardia são características e dificilmente confundidas com demais patologias (BRASIL, 2015).

Entre os anos de 2006 e 2016 a houve semelhança entre as porcentagens de sífilis gestacional nas regiões do Brasil. Na região Sul houve 2.235 casos, na região Norte 1.915 casos, na região Nordeste 3.518 casos, na região Sudeste 7.230 casos e na região Centro-Oeste 1.542 casos, no estado de São Paulo de 3.598 casos e na capital de São Paulo de 1.512 casos (BRASIL, 2020; BOTTURA BR, et al., 2019).

A formas de sífilis é de notificações compulsória, possuindo a obrigatoriedade a sua realização por profissionais da saúde, sendo que a sua falta de cumprimento confere infração à legislação de saúde (LAFETÁ KTG, et al., 2016). Mas, mesmo com uma punição clara, a subnotificação é frequente, a exemplo estudos realizados em Palma (TO) e no Estado de São Paulo, que demonstrou o triplo de casos identificados, em comparação com os notificados (CAVALCANTE PA, et al., 2017; MASCHIO-LIMA T, et al., 2019)

Considerando o impacto da sífilis congênita na assistência em saúde pública e a necessidade de seu controle, o presente estudo possui o objetivo de avaliar o perfil epidemiológico dos casos notificados de Sífilis Congênita no munícipio de Porto Velho entre os anos de 2010-2020, para que possamos definir medidas intervencionistas efetivas.

\section{MÉTODOS}

Trata-se de um descritivo, quantitativo dos casos notificados de sífilis congênita no município de Porto Velho, estado de Rondônia, realizado com base nos dados disponibilizados no período de janeiro de 2010 a dezembro de 2020. Utilizado como critério de inclusão a população composta por todos os casos de sífilis congênita notificado no referido munícipio e no período estabelecido de acordo com a Classificação Internacional de Doenças (CID 10) codificada como A50. Dados referentes a anos anteriores a 2010 foram excluídos do presente artigo.

Para a captação dos dados de casos de sífilis congênita, utilizou-se a plataforma do departamento de informática do Sistema Único de Saúde do Brasil (DATASUS), órgão responsável por coletar informações sobre a saúde no Brasil. A plataforma digital foi escolhida pelo cenário atual causado pela pandemia do COVID-19, fato este que impossibilita a pesquisa de campo.

O levantamento dos dados foi baseado em pesquisas da plataforma do DATASUS através do Sistema de Informação de Agravos de Notificações (SINAN), foram incluídos todos os casos de sífilis em crianças, notificados no município de Porto Velho no período de janeiro de 2010 a dezembro de 2020. As variáveis dos estudos foram: sociodemográficos (faixa etária materna, escolaridade materna, cor da pele e local de residência); diagnóstico (realização do pré-natal e quando foi feito o diagnóstico materno, pré-natal, parto ou após o parto); tratamento da gestante (adequado, inadequado, não realizado ou ignorado); diagnóstico da criança; e a evolução dos casos.

Baseado no supracitado foram analisados 973 casos confirmados de mulheres a partir dos 10 anos de idade, sendo usado como fator de seleção a idade mínima com maior prevalência de gravidez. Os dados foram tabulados e para a representação gráfica e análise dos dados foram utilizados os programas Microsoft Excel 2016 e o Microsoft Word 2016.

Por se tratar de dados secundários de domínio público, o estudo não necessitou de apreciação por Comitê de Ética em Pesquisa, sendo seguidas as normas preconizadas pelo Conselho Nacional de Saúde em sua Resolução CNS no 466, de 12 de dezembro de 2012. 


\section{RESULTADOS}

O estudo foi delimitado segundo os casos notificados de 2010 até 2020 entre crianças de 7 a 27 dias. No período de 2010 a 2020, no município de Porto Velho, foram notificados 537 casos. Dentre estes anos de notificação, o ano de maior episódio foi no ano de 2018 (87 casos notificados), totalizando 16\% dos casos. O segundo ano com maior número de casos foi o ano de 2017, com 85 casos e porcentagem 15,9\%. Entre os anos apenas o ano de 2018 o diagnóstico de sífilis não foi recente, evoluindo para natimorto (Tabela 1).

Tabela 1 - Casos de sífilis congênita segundo idade da criança por ano de diagnóstico.

\begin{tabular}{llllllllllll}
\hline Idade da Criança & \multicolumn{10}{c}{ Ano } \\
\cline { 2 - 11 } & $\mathbf{2 0 1 0}$ & $\mathbf{2 0 1 1}$ & $\mathbf{2 0 1 2}$ & $\mathbf{2 0 1 3}$ & $\mathbf{2 0 1 4}$ & $\mathbf{2 0 1 5}$ & $\mathbf{2 0 1 6}$ & $\mathbf{2 0 1 7}$ & $\mathbf{2 0 1 8}$ & $\mathbf{2 0 1 9}$ & $\mathbf{2 0 2 0}$ \\
\hline Menos de 7 dias & 7 & 20 & 26 & 44 & 55 & 66 & 64 & 84 & 87 & 49 & 26 \\
$\mathbf{7}$ a 27 dias & - & - & 1 & - & - & 2 & - & 1 & - & - & 2 \\
$\mathbf{2 8}$ dias a 364 dias & - & - & - & - & - & - & - & 1 & -- & 2 & - \\
$\mathbf{1}$ ano & - & - & - & - & - & - & - & - & - & - & -
\end{tabular}

Fonte: Kisner JGM, et al., 2021; dados extraídos de MS/SVS/Departamento de Doenças de Condições Crônicas e Infecções Sexualmente Transmissíveis.

A Tabela 2 apresenta a distribuição dos indicadores da sífilis em gestantes. Ao analisar o estado social da mãe, a faixa etária mais prevalente de mães com filhos portadores de sífilis foi a de 20 a 29 anos, possuindo o total de $53,4 \%$ dos casos no intervalo de 10 anos. Vale ressalta o elevado número de 121 casos (23\%) entre a 15 a 19 anos, idade precoce para todos os cuidados que um filho acarreta.

Tabela 2 - Características sociodemográficas dos casos de Sífilis congênita notificados no município de Porto Velho, no período de $2010-2020$

\begin{tabular}{|c|c|c|}
\hline Características & $\mathbf{N}$ & $\%$ \\
\hline \multicolumn{3}{|l|}{ Faixa Etária } \\
\hline 10 a 14 anos & 11 & 2 \\
\hline 15 a 19 anos & 121 & 22 \\
\hline 20 a 29 anos & 281 & 52 \\
\hline 30 a 39 anos & 106 & 19,7 \\
\hline 40 anos ou mais & 13 & 2,4 \\
\hline Ignorado & 5 & 0,9 \\
\hline \multicolumn{3}{|l|}{ Raça } \\
\hline Ignorado/Branco & 37 & 6,9 \\
\hline Branca & 47 & 8,8 \\
\hline Preta & 25 & 4,6 \\
\hline Parda & 424 & 79,4 \\
\hline Indígena & 1 & 0,1 \\
\hline \multicolumn{3}{|l|}{ Escolaridade da Mãe } \\
\hline Analfabeto & 3 & 0,5 \\
\hline $1^{\mathrm{a}}$ a $4^{\mathrm{a}}$ série incompleta & 54 & 9,5 \\
\hline $4^{\mathrm{a}}$ série completa & 29 & 5,1 \\
\hline $5^{\underline{a}}$ a $8^{\underline{a}}$ série incompleta & 157 & 27,7 \\
\hline \multicolumn{3}{|l|}{ Escolaridade } \\
\hline Fundamental Completo & 32 & 5,6 \\
\hline Médio Incompleto & 77 & 12,7 \\
\hline Médio Completo & 82 & 14,5 \\
\hline Superior Incompleto & 10 & 1,7 \\
\hline Superior Completo & 3 & 0,5 \\
\hline Não se aplica & 1 & 0,1 \\
\hline Ignorado & 117 & 20,7 \\
\hline
\end{tabular}

Fonte: Kisner JGM, et al., 2021; dados extraídos de MS/SVS/Departamento de Doenças de Condições Crônicas e Infecções Sexualmente Transmissíveis. 
O grau de instrução da mãe que tiveram filhos com sífilis congênita durante o período estabelecido, os números que sobressaíram foram referentes ao ensino fundamental incompleto (27\%) e os ignorados que representam $20 \%$ dos casos.

Quanto aos antecedentes epidemiológicos gestantes, foram verificados que 404 casos $(75,2 \%)$ realizaram o pré-natal e 265 (50\%) foram diagnosticados neste período. Entretanto, $199(37 \%)$ o diagnóstico foi realizado no momento do parto/curetagem.

Tabela 3 - Características do diagnóstico dos casos de Sífilis congênita notificados no município de Porto Velho, no período de 2010-2020.

\begin{tabular}{lll}
\hline Características & N & $\%$ \\
\hline Realização de pré-natal & & \\
\hline Sim & 404 & 75,2 \\
Não & 116 & 21,6 \\
Ignorado & 17 & 3,1 \\
\hline Momento do diagnóstico da sífilis & & \\
materna & 265 & 49,1 \\
\hline Durante o pré-natal & 199 & 36,9 \\
No momento do parto/curetagem & 58 & 10,7 \\
Após o parto & 4 & 7,4 \\
Não realizado & 13 & 2,4 \\
Ignorado & & \\
\hline Esquema de tratamento materno & 29 & 5,4 \\
\hline Adequado & 345 & 64,2 \\
Inadequado & 143 & 26,6 \\
Não realizado & 20 & 3,7 \\
lgnorado & & \\
\hline
\end{tabular}

Fonte: Kisner JGM, et al., 2021; dados extraídos de MS/SVS/Departamento de Doenças de Condições Crônicas e Infecções Sexualmente Transmissíveis.

Das gestantes infectadas por sífilis apenas $5 \%$ realizaram o tratamento de forma adequado, $26,6 \%$ não realizaram tratamento e $64,2 \%$ realizaram o tratamento inadequado.

\section{DISCUSSÃO}

O estudo apresentou elevada ocorrência de sífilis congênita no município de Porto Velho. A Organização Pan-Americana de Saúde (OPAS) e do Ministério da Saúde (MS) instituíram metas para reduzir a incidência de sífilis congênita no Brasil, estás metas estão longe de serem alcançadas (BRASIL, 2015). O pacto está palpado em eliminar a doença, definindo como meta reduzir a incidência da sífilis congênita para menos de 0,5 casos/1.000 nascidos vivos. Até 2018 os casos notificados em Porto Velho quadruplicaram em relação a 2011. Em 2019 o município contabilizou 9.286 nascidos vivos e 49 casos de sífilis congênita, resultando em 5,27 casos/1.000 nascidos vivos, no ano de 2018 fica ainda mais assustador, resultando em 8,9 casos/1.000 nascidos vivos (BRASIL, 2021).

Outro estudo realizado no estado do Acre nos anos de 2009-2018 demostrou um aumento linear de casos notificados (BRANCOT JT, et al., 2020). No estado de São Paulo e na cidade de Palmas também registraram aumento linear nos casos notificados. Em Palmas as taxas de casos notificados ano a ano aumentaram de 2,9/1.000 nascidos vivos no ano de 2007 para 8,1/1.000 nascidos vivos em 2014 (CAVALCANTE PA, et al., 2017). No estado de São Paulo houve um aumento linear e constante de 1,4 casos por 1.000 nascidos vivos no ano de 2007 par a 5,8 por 1.000 nascidos vivos em 2016 (MASCHIO-LIMA T, et al., 2019). Estes estudos demostram o quando a quantidade de casos está muito acima da meta estabelecida

Taxas de incidência em estudos internacionais sobre a sífilis congênita também apresentam aumento nos casos notificados. No Canadá em 2014 a taxa de sífilis congênita era de 0,3 casos por 100.000 nascidos 
vivos, passando para 1,5 casos por 100 mil nascidos vivos em 2015 (CHOUDHRI Y, et al., 2018). Na Inglaterra, de janeiro de 2011 a dezembro de 2016, as taxas de casos notificados de sífilis congênita aumentaram 1,1 para 2,3 casos por 100.000 habitantes (FUREGATO M, et al., 2017). Porém, o número total de casos notificados em todo mundo caiu de 748.000 em 2012 para 661.000 em 2016, caindo também o de 539 para 473 casos por 100 mil nascidos vivos. A maioria dos casos notificados no estudo ocorreu na região africana, possuindo o 62\% dos casos em 2012 e 61\% em 2016 (KORENROMP EL, et al., 2019)

O aumento de número de notificações no presente artigo pode ser atribuído não somente ao número de casos que se multiplicaram, mas também à melhoria, e intensificação, das ações de vigilância epidemiológica pelas secretárias de saúde, diminuindo o número de subregistros (ANDRADE HS, et al., 2019; DIAS APS, et al., 2018). O aumento também pode ser respaldado pela facilitação do diagnóstico, atribuídos aos testes rápidos e a capacitação dos profissionais de saúde, além da ampliação da cobertura do pré-natal devido a implantação das equipes da Estratégia Saúde da Família (BRASIL, 2021; ANDRADE HS, et al., 2018)

Analisando as características sociodemográficas dos casos de sífilis congênita no município de Porto Velho constata-se que $98,5 \%$ (529) dos casos foram diagnosticados até o sétimo dia de vida, dado similar no estado do Acre com 96,2\% (580) no período de 2009 a 2018, isto manifestar que o diagnóstico de sífilis congênita ocorreu no tempo oportuno, facilitando o início do tratamento precoce (BRANCOT JT, et al., 2020).

Quando se análise o perfil materno os resultados mostram que a faixa etária de 20 a 29 anos é a mais suscetível ao acometimento pela enfermidade, representando $52 \%$ dos casos notificados, esta faixa etária também foi identificada na maioria dos estudos (BRANCOT JT, et al., 2020; MARQUES JVS, et al., 2018; SOUZA BS de O, et al., 2018), no estado de São Paulo a faixa etária de 20 a 29 também foi a mais prevalente, apresentando um percentual de $51,30 \%$ no período de 2007 a 2016, e no estado Acre a mesma faixa etária obteve um incidência de 289 (47,9\%) no período de 2009 a 2018, esta faixa etária predomina por representar o auge da fase reprodutiva (BRANCOT JT, et al., 2020; BOTTURA BR, et al., 2019).

A segunda faixa etária que apresenta o maior número de casos notificados é a de 15 a 19 anos, apresentando 22\% (121) dos casos notificados, este dado preocupante, pois demonstra um número expressivo de adolescentes não utilizando preservativos na relação sexual (SOUZA WN, 2017; LAGO EG, 2016).

Segundo a escolaridade é nítido a relação entre a baixa escolaridade e exposição da doença, possuindo um total de $189(33,4 \%)$ casos com mães que possuem o ensino fundamental incompleto ou completo (da $5^{\underline{a}}$ a $8^{\text {a }}$ série). Cenário similar foi encontrado no Acre, onde $45,5 \%$ das mães possuem o ensino fundamental completo ou incompleto. A escolaridade corrobora com uma série de fatores limitantes no processo saúdedoença, ou seja, indicadores de saúde melhoram com a escolarização e boa saúde melhora o desempenho em tarefas escolares. Apesar do elevado número de subregistro, é inquestionável que a baixa escolaridade possui uma relação com ao limitado conhecimento sobre medidas de prevenção. (CAVALCANTE PA, et al., 2017).

A transmissão vertical da sífilis ocorre a partir da 9aㅗ semana de gestação, entretanto é mais frequente entre a $16^{\underline{a}}$ e $28^{a}$ semanas, este fato reforça que o diagnóstico e tratamento precoce é essencial para barrar o aumento das taxas de incidência da sífilis congênita (CARDOSO ARP, et al., 2018). Adiciona-se a isso, a identificação tardia da doença em gestantes se relaciona ao pior prognóstico para o termino do tratamento em tempo hábil (SOUZA BS de O, et al., 2018).

Protocolos clínicos e diretrizes terapêuticas para a prevenção da transmissão vertical de doenças sexualmente transmissíveis, como HIV, sífilis e hepatites virais, recomenta o diagnóstico seja realizado por dois tipos de testes, o primeiro são os testes não treponêmicos (teste rápido ou FTA-Abs ou TPHA ou EQL ou ELISA) e o segundo são os testes não treponêmicos (VDRL ou RPR ou TRUST), isto é feito para que assim possa descartar reação cruzar pela gravidez ou outras infecções (BRASIL, 2019).

Todos os recém-nascidos de mães com diagnóstico de sífilis devem ser investigados para sífilis congênita. Gestantes que foram tratadas adequadamente pode ser realizado apenas o teste não treponêmica (VDRL) no recém-nascidos, caso o teste venha negativo, o neonato deve ser acompanhado, existindo a 
impossibilidade do seguimento realiza-se tratamento com dose única de penicilina G Benzatina. Em outros casos deve ser realizado a investigação com VDRL, radiografia de ossos longos, hemograma e exame de líquor, o tratamento será definido de acordo com os resultados dos exames realizados. Após isso, a criança deve realizar acompanhamento com o pediatra por consultas mensais até o 60 mês e, em seguida, a cada dois meses até o primeiro ano de vida, VDRL deve ser trimestral e exame de líquor a cada seis meses, até que ele esteja normalizado (LAFETÁ KTG, et al., 2016).

Apesar do elevado número de gestantes ter realizado o pré-natal $(75,2 \%)$, muitas foram diagnósticas com a sífilis apenas no momento do parto ou curetagem (36,9\%). Esse fato evidencia que, apesar do acompanhamento médico ou da enfermagem, ainda persiste, no país, o diagnóstico tardio da infecção (CARDOSO ARP, et al., 2018, LAFETÁ KTG, et al., 2016). Adiciona-se a isso, que o diagnóstico tardio se relaciona ao pior prognóstico para conclusão do tratamento em tempo hábil, para a prevenção da transmissão vertical. Entretanto, estudos no Reino Unido e no Sul da Itália obtiveram taxas menores que 0,02 casos/1.000 nascidos vivos durante o período de 2010 a 2015 (SIMMS I, et al, 2017).

Em vista disso, a vigilância do agravo deve ser realizada no período em que a intervenção ainda é possível. O tratamento adequado da sífilis é feito com a penicilina G Benzatina, única opção segura e eficaz para o tratamento, outros antibióticos, como as tetraciclinas orais e os macrolídeos, são utilizados para o tratamento em adultos, mas não são recomendados no período da gestação por causa da toxicidade ao feto ou por não atravessarem a barreira placentária. Deve ser buscar a comunicação com os parceiros sexuais para testagem e tratamento adequado em momento oportuno (BRASIL, 2019)

O tratamento inadequado é comum, como o mostra o estudo, onde $64,2 \%$ das gestantes não realizaram o tratamento adequado. Muitas vezes, o tratamento inadequado é atribuído à falha no serviço em convocar o parceiro e realizar a terapêutica (LAFETÁ KTG, et al., 2016). A terapia do parceiro é de extrema relevância para o sucesso do tratamento da sífilis durante a gestação. Somado a isto, nos anos de 2014 a 2016 o Brasil passou por uma situação grave vulnerabilidade sanitária devido à ausência de plantas farmoquímicas para a produção de antibióticos por fermentação e consequentemente a dependência de importações de matériaprima, gerando uma falta de penicilina (RODRIGUES PHA, et al., 2018).

O esforço unificado da assistência pré-natal de qualidade, exames laboratoriais em tempo hábil, tratamento adequado do casal e a conscientização de todos envolvidos, será possível o objetivo estabelecido pela OPAS e MS (DOMINGUES RM, 2016). A utilização da Estratégia Saúde da Família é essencial para a aproximação da população, devido a ser uma porta de entrada para os serviços de saúde e a presença de agentes comunitários de saúde, para a intervenção na educação continuada (LAFETÁ KTG, et al., 2016).

A sífilis congênita é uma doença que pode ser prevenida com a simples implementação de estratégias efetivas de diagnóstico precoce e tratamento de sífilis nas gestantes, sendo algo intrínseco da assistência à saúde materno-infantil (BRASIL, 2019). Sendo assim, o aumento da incidência de casos notificados no município é indicativo de falha na atenção do pré-natal.

\section{CONCLUSÃO}

Nota-se as vulnerabilidades intrínsecas aos casos notificados de sífilis como escolaridade e qualidade da atenção à saúde. O estudo demostrou que a maioria das gestantes infectadas possuem de 20 a 29 anos, com baixa escolaridade e mesmo que tenha sido diagnóstica durante o pré-natal, uma grande parcela só teve o diagnóstico no parto ou curetagem, soma-se a isso a existência da prevalência do tratamento inadequado e a persistência do não tratamento dos parceiros. Isto fomenta a necessidade de maior integração dos serviços envolvidos e a criação de políticas públicas gravídico-puerperal para que enfim o agravo causado pela sífilis tenha uma redução ou, no mundo ideal, a extinção de casos.

\section{REFERÊNCIAS}

1. ANDRADE ALMB, et al. Diagnóstico tardio de sífilis congênita: uma realidade na atenção à saúde da mulher e da criança no Brasil. Revista Paulista de Pediatria, 2018; 36(3): 376-381. 
2. ANDRADE HS, et al. Caracterização epidemiológica dos casos de sífilis em mulheres. Ciência \& Saúde, 2019; 12(1): 32124

3. ALVES P, et al. Evolução temporal e caracterização dos casos de sífilis congênita em Minas Gerais, Brasil, 20072015. Ciência \& Saúde Coletiva [online]. 2020; 25(8): 2949-2960.

4. ALZATE-GRANADOS JP, et al. Disparidades en la incidencia de sífilis congénita en Colombia 2005 a 2011 : Un estudio ecológico. Rev de Salud Publica, 2012; 14(6): 965-977.

5. BOTTURA BR, et al. Perfil epidemiológico da sífilis gestacional e congênita no Brasil - período de 2007 a 2016. Arquivo Médico Hospitalar da Faculdade de Ciência Médicas da Santa Casa, 2019; 64(2): 69-75

6. BRANCOT JT, et al. Perfil epidemiológico dos casos notificados de sífilis congênita no estado do Acre nos anos de 2009-2018. Revista Eletrônica Acervo Saúde, 2020; 12(9): e4347.

7. BRASIL, Ministério da Saúde. Disponível em http://www.datasus.gov.br. Acessado em 02 de junho de 2020.

8. BRASIL. Ministério da Saúde. Disponível em: https://portalsinan.saude.gov.br/. Acessado em 28 de fevereiro de 2021.

9. BRASIL. Ministério da Saúde. $2015 . \quad$ Disponível http://conitec.gov.br/images/Consultas/Relatorios/2015/Relatorio_Penicilina_SifilisCongenita_CP.pdf. Acessado em: 25 de maio de 2021.

10. BRASIL. Ministério da Saúde. 2016. Disponível em: http://www.aids.gov.br/pt-br/pub/2016/manual-tecnico-paradiagnostico-da-sifilis. Acessado em: 10 de junho de 2021.

11. BRASIL Ministério Saúde. bvsms.saude.gov.br/bvs/publicacoes/guia_vigilancia_saude_3ed.pdf .> Acesso em: 28 de maio de 2021

12. BRASIL. Ministério da Saúde. 2019. Disponível em http://www.aids.gov.br/pt-br/pub/2019/boletim-epidemiologicosifilis-2019. Acessado em 02 de junho de 2021

13. BRASIL. Ministério da Saúde. 2015. Disponível em: <http://www.aids.gov.br/pt-br/pub/2015/protocolo-clinico-ediretrizes-terapeuticas-para-atencao-integral-pessoas-com-infeccoes.> Acessado em: 28 fevereiro de 2021.

14. BRASIL. Ministério da Saúde. 2019. Disponível em: http://www.aids.gov.br/pt-br/pub/2015/protocolo-clinico-ediretrizes-terapeuticas-para-prevencao-da-transmissao-vertical-de-hiv. Acessado em: 06 junho de 2021.

15. BRASIL. Ministério da Saúde. 2020. Disponível em: http://www.aids.gov.br/pt-br/pub/2015/protocolo-clinico-ediretrizes-terapeuticas-para-atencao-integral-pessoas-com-infeccoes. Acessado em: 10 de junho de 2021.

16. CARDOSO ARP, et al. Análise dos casos de sífilis gestacional e congênita nos anos de 2008 a 2010 em Fortaleza, Ceará, Brasil. Ciência \& Saúde Coletiva, 2018; 23(2): 563-574

17. CAVALCANTE PA, et al. Sífilis gestacional e congênita em Palmas, Tocantins, 2007-2014.Epidemiologia e Serviços de Saúde, 2017; 26(2): 255-264.

18. CHOUDHRI Y, et al. Sífilis infecciosa e congênita no Canadá, 2010-2015. Can Comm Dis Rep., 2018; 44(2): 43-48.

19. DIAS APS, et al. A sífilis no atual cenário brasileiro. Health Research Journal, 2018; 1(2): 1-21.

20. DOMINGUES RM, LEAL MC. Incidência de sífilis congênita e fatores associados à transmissão vertical da sífilis: dados do estudo Nascer no Brasil. Cad Saúde Pública [Internet], 2016; 32(6): e00082415.

21. FUREGATO M, et al. Fatores associados a quatro casos atípicos de sífilis congênita na Inglaterra, 2016 a 2017 : uma análise ecológica. Euro Surveill., 2017; 22(49): 17-00750.

22. KORENROMP EL, et al. Global burden of maternal and congenital syphilis and associated adverse birth outcomesestimates for 2016 and progress since 2012. PLoS One, 2019; 14(2).

23. LAFETÁ KR, et al. Maternal and congenital syphilis, underreported and difficult to control. Rev Bras Epidemiol., 2016; 19(1): 63-74.

24. LAGO EG. Current perspectives on prevention of mother-to-child transmission of syphilis. Cureus, 2016; 8(3): e525.

25. MARQUES JVS, et al. Perfil Epidemiológico da Sífilis Gestacional: Clínica e Evolução De 2012 A 2017. Revista Sanare, 2018; 17(02): 13-20.

26. MASCHIO-LIMA T, et al. Epidemiological profile of patients with congenital and gestational syphilis in city in the State of São Paulo, Brazil. Revista Brasileira de Saúde Materno Infantil, 2019; 19(4): 865-872.

27. RODRIGUES AR, et al. Atuação de enfermeiros no acompanhamento da sífilis na atenção primária. Rev Enferm UFPE, 2016; 10(4): 1247-55.

28. RODRIGUES PHA, et al. A evolução recente da indústria farmacêutica brasileira nos limites da subordinação econômica. Physis: Revista de Saúde Coletiva, 2018; 28(1): 1-22.

29. SIMMS I, et al. The incidence of congenital syphilis in the United Kingdom: February 2010 to January 2015. BJOG, 2017; 124: 72-77.

30. SOUZA BS de O, et al. Análise epidemiológica de casos notificados de sífilis. Revista da Sociedade Brasileira de Clínica Médica, 2018; 16(2): 94-98.

31. SOUZA WN, BENITO LA. Perfil epidemiológico da sífilis congênita no Brasil no período de 2008 a 2014. Universitas: Ciências da Saúde, 2016; 14(2): 1-8.

32. SUTO CSS, et al. Assistência pré-natal a gestante com diagnóstico de sífilis. Revista de Enfermagem e Atenção à Saúde, 2016; 5(2): 18-33

33. WORLD HEALTH ORGANIZATION (WHO). The global elimination of congenital syphilis: rationale and strategy for action [Internet]. Geneva: Switzerland; 2007. Available from: http:// apps.who.int/iris/bitstream/10665/43782/1/9789241595858_eng.pdf 